\title{
Fractal-Shaped Metamaterials and Applications to Enhanced-Performance Devices Exhibiting High Selectivity
}

\author{
He-Xiu Xu, Guang-Ming Wang, and Chen-Xin Zhang \\ Department of Electromagnetic Field and Microwave Technique, Missile Institute of Air Force Engineering University, \\ 713800 Sanyuan, China \\ Department of Electromagnetic Field and Microwave Technique, Missile Institute of Air Force Engineering University, 713800 Sanyuan, \\ China
}

Correspondence should be addressed to He-Xiu Xu, hxxu20008@yahoo.cn

Received 16 February 2011; Accepted 31 May 2011

Academic Editor: Lei Zhu

Copyright (C) $2012 \mathrm{He}$-Xiu Xu et al. This is an open access article distributed under the Creative Commons Attribution License, which permits unrestricted use, distribution, and reproduction in any medium, provided the original work is properly cited.

\begin{abstract}
Novel single negative metamaterial (MTM) transmission lines (TLs) are presented and studied in microstrip technology. They consist of a host TL in the conductor strip and a fractal-shaped complementary ring resonator (CRR) etched in the ground plane. Two types of fractal-shaped CRR are involved including the Moore and Hilbert. It is found that fractal perturbation in CRR results in lower and more transmission zeros in comparison with conventional CRR using nonfractal geometries. The single negativepermeability or -permittivity of these MTM TLs which associated with the resultant multitransmission zeros occurs by turns and should benefit devices with high selectivity requirement. Potential application of these MTM cells are illustrated by two examples, one is the microstrip stepped-impedance transformers (SIT) operating at $3.5 \mathrm{GHz}$ with two edged attenuation poles to introduce selectivity; the other one is the Hi-Lo microstrip low-pass filter (LPF) with cutoff frequency $2.5 \mathrm{GHz}$ exhibiting improved selectivity $(77.3 \mathrm{~dB} / \mathrm{GHz})$. By constructing the low-impedance sections as hybrid prefractal shape and crown square, both the SITs and LPF obtained additional bandwidth enhancement and good matching. Consistent results between simulation and measurement have confirmed the design concept.
\end{abstract}

\section{Introduction}

Complementary split ring resonators (CSRRs), firstly exploited by Falcone et al. in 2004 [1, 2], are frequently loaded sub-wavelength particles in contribution to negative permittivity of metamaterials (MTMs) [3-6]. These CSRRs-loaded left-handed (LH) MTMs allow the signal to transmit freely due to the double negative permittivity $(\varepsilon)$ and permeability $(\mu)$ while to inhibit signal propagation due to single negative$\varepsilon$ or $-\mu$.

As exposed to fractal geometry, it combined with EM theory has led to a new concept for circuits and antennas design. Currently, fractals combined with LH MTMs have become a heated topic, for instance, fractal perturbation in CSRRs results in a significant lower resonance $[7,8]$, multiband behavior [9], and broadband performance [10] of the LH cell. Others also proposed Hilbert curve inclusions for the engineered artificial magnetic materials [11], and the authors even exploited fractal concept in UWB filter for elevation of passband performance [12].

Up to now, most research focuses on the double negative MTMs issue, few literatures are reported concerning the use of single negative- $\varepsilon$ or negative- $\mu$ to improve the selectivity $[5,6]$. On the other hand, integrated concept has been poured into the implementation of antenna such as a balun combining a dipole antenna [13], which should be extremely beneficial to the low cost and miniaturized circuit size.

In view of them, the body of this work aims to develop high-performance devices integrated with single negative MTMs to pursue high selectivity and simultaneously extend the concept of [12] to this work for passband performance enhancement. To this end, novel miniaturized MTM cells employing fractal-shaped complementary ring resonator (CRR) are proposed and exploited. Unlike CSRRs with their axes parallel to the vector of electric field (E-field) [16], novel fractal-shaped CRR is with much complexity, for 
example, Moore-shaped CRR (M-CRR) demonstrated with superior magnetic-field (H-field) parallel to the axes of CRR thus exhibiting negative- $\mu$ while one type of Hilbert-shaped CRR (H-CRR) with axial superior E-field thus exhibiting negative- $\varepsilon$ beyond the fundamental resonance. In some cases, the negative- $\mu$ or $-\varepsilon$ even occurs by turns in higher bands. The paper is organized as follows.

In Section 2, revised Moore and Hilbert fractal curves of different operation are presented. By etching these curves in the ground plane, symmetric M-CRR and asymmetric HCRR with controllable resonance are developed. Then MTM unit cells employing M-CRR and $\mathrm{H}-\mathrm{CRR}$ are proposed and investigated in depth in terms of multiband behavior and miniaturization. The relation between transmission zeros and number of initial generator is also discussed and derived. Following that, the working mechanism of fractal-shaped CRR is investigated in terms of field distribution. Some interesting conclusions are also made. In Section 3, the effective complex constitutive EM parameters of M-CRRand H-CRR-loaded MTM cells are extracted. These results are in good agreement with ones predicted based on the analysis of EM field distribution. In Section 4, maximal flat stepped-impedance transformers (SITs) using M-CRR and Hi-Lo microstrip low-pass filter (LPF) using H-CRR are provided. Influence of hybrid prefractal geometry on performance of SITs is also studied. Crown square perturbed in the upper conductor strip of LPF is exploited in terms of enhanced in-band behavior. Consistent simulated and measured results are given. Finally, a major conclusion is highlighted in Section 5.

\section{Proposed MTM Unit Cells}

In this section, fractal perturbation is firstly exploited in CRR. The most unlikeness of CRR to CSRRs lies in whether a split gap is adopted. Since the additional characteristic is required, any fractal curves with closed configuration are beneficial to this design other than Peano [14], Hilbert [15], or Wunderlich [16] fractals which feature open curves and have ends on the opposite sides. Note that CRR can be straightforwardly extended to complementary ring resonators (CRRs); however, it is worth to point out that the resultant concentric two- or multiring resonators brought another issue of much complexity when regarding fractal implementation. What is more, a tradeoff is demanded between the insertion length and the gap between two neighboring CRR.

\subsection{Revised Space-Filling Curves Based on Moore and Hilbert.} Fractal geometry, originally proposed by Mandelbrot to describe a family of recursively generated irregular fragments, possesses an inherent self-similarity and a fractional dimension. It is characterized by two factors, that is to say, the iteration factor (IF) and the iteration order (IO). The IF represents the construction law of initial generator while the IO predicts how many iteration processes are carried out. In this section, two sets of revised structures are involved based on Moore and Hilbert fractal curves.

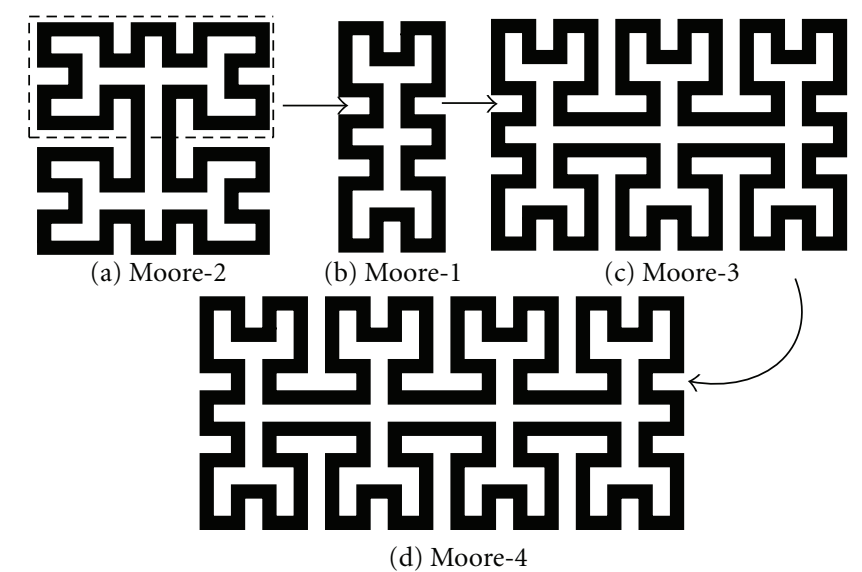

FIGURE 1: Flowchart of the proposed fractals based on Moore spacefilling curve.

The second iteration of Moore space-filling curve, denoted as Moore-2 for convenience in this work, was adopted in design of miniaturized antenna [16] and rat-race coupler [17]. It composes two fundamental elements (in dashed frame) and can be considered as a symmetric structure. Unlike [17], the joint segment between two elements is length of the minimal fractal segment. We denote the fundamental element as Moore-1 for simplicity. By merging $n$ copies of Moore-1 and inserting one minimal fractal segment in the joint between two adjacent elements, novel fractal structure of Moore- $n$ is developed. Figure 1 illustrates the flowchart of the proposed fractals based on Moore for higher operation.

The resultant Moore fractals mentioned above is from the point of best area-filling efficiency. Number of resultant fractal segments of engineered Moore- $n\left(N_{n}\right)$ is determined by

$$
N_{n}=n N_{1}
$$

and total length $L^{n}$ extends with each operation and is calculated as

$$
L^{n}=\frac{8 n}{2 n+3} L_{0}^{n}
$$

where $N_{1}$ is the number of fractal segments of Moore-1 and $L_{0}^{n}$ is the perimeter of conventional rectangle occupying the same area with its corresponding Moore- $n$. Consulting (1), we find that the fractal segments are linearly increased, which would be extremely useful to current fractals other than conventional one with exponentially incremental fractal segments. Accordingly, the perimeter of the engineered Moore- $n$ is also linearly proportional to the higher operation order $n$.

Conventional Hilbert curves are particularly interesting for further miniaturization due to their strong capability of space-filling. It is generated in an iterative fashion with the use of collinear transformations, which consist of a continuous line connecting the centers of a uniform background grid. Suppose the curve filled in a square section of 


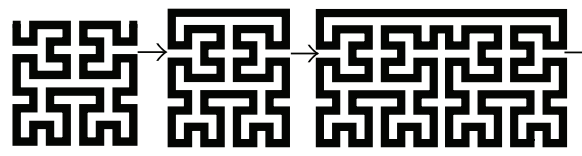

(a) Hilbert (b) Hilbert-1

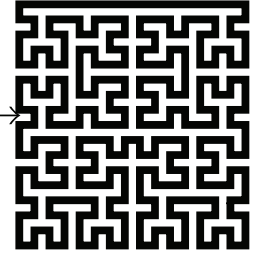

(d) Hilbert-4

Figure 2: Flowchart of the proposed fractals based on Hilbert space-filling curve.

$S$ as external side. By increasing the iteration level, the space between lines diminishes accordingly and the length of total perimeter increases as

$$
L(n)=\left(2^{n}+1\right) S \text {. }
$$

From (3), the perimeter is exponentially increased with the incremental $n$.

Although conventional Hilbert curves are convenient for application in structures such as end-coupled microstrip resonators [15], they are not directly applicable to current close-loop design. In this regard, a transformation is required. Hilbert curve of second iteration order, with configuration shown in Figure 2, is characterized by a fractal dimension of approximate 2 according to $D=\ln 63 / \ln 8$. By extending the start and the end point of it by length of the minimal fractal segment, and then joining the terminals with an additional line, the resultant fundamental Hilbert-1 is constructed. With the basic generator, Hilbert- $n$ is envisaged by uniting $n$ copies of Hilbert- 1 in proper placement to form a ring resonator. Note that resultant Hilbert- $n$ is an asymmetric structure with total path length approximately linear proportion to operation order $n$.

In conclusion, both revised spacing-filling curves have an unoccupied area in their center, which should be beneficial to excitation of the MTM cell. According to effective medium theory, the wavelength should be much larger than the dimension of constituent elements that form the medium. MTM unit cell based on Hilbert-3 is too large to be considered as homogeneous medium. As to Hilbert-4, we considered expanding four Hilbert elements in two orientations to reduce dimensions in transmission orientation. For the same causation, the MTM cell based on Moore is confined to Moore- 5 in the following section.

2.2. Unit Cells That Use M-CRR. In this section, microstrip MTM unit cells based on M-CRR are firstly investigated in terms of multiresonances (multitransmission zeros) and miniaturization. The influence of fractals to the loaded and unloaded quality factor is less interesting in this work and can be found in many other works. Layout of presented MCRR-loaded MTM cell is depicted in Figure 3. Additional unlikeness to the CSRRs-loaded resonant-type LH unit cell is that there is no capacitive gap in the upper conductor strip providing the effective negative permeability.

In order to provide a deep insight into the influence of different operations to the resonance performance, five

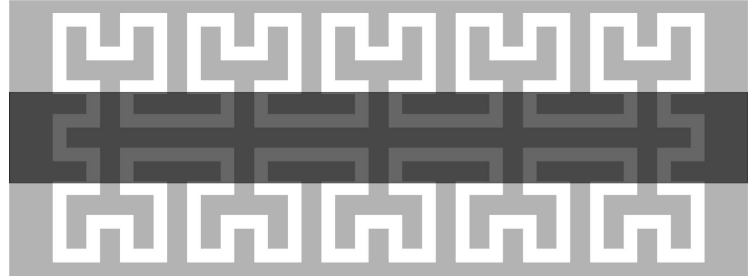

FIgURE 3: Microstrip MTM unit cell using Moore-5. Note that the M-CRR (depicted in white) is etched in the ground plane (depicted in light grey) underneath the host microstrip line (depicted in dark grey).

MTM units with Moore fractal up to Moore- 5 are simulated on a $1 \mathrm{~mm}$ thick F4B-2 substrate with dielectric constant of 2.65 and dielectric loss tangent of 0.001 . For comparison, analysis of conventional microstrip structure applying rectangle CRR occupying the same area with Moore-5 is also performed. Length and width of the minimal fractal segment of CRR are set to be $1 \mathrm{~mm}$ and $0.4 \mathrm{~mm}$, respectively, for all cases. Meanwhile, width of microstrip line is tailored to $2.7 \mathrm{~mm}$ referring to the termination impedance $50 \Omega$. Dimensions of Moore- 1 to Moore- 5 are listed as $3.4 \mathrm{~mm}$, $7.4 \mathrm{~mm}, 11.4 \mathrm{~mm}, 15.4 \mathrm{~mm}$, and $19.4 \mathrm{~mm}$, respectively. The MOM-based EM simulator Ansoft Designer is adopted for simulation.

Figure 4 illustrates the obtained S-parameters of these microstrip MTM cells in a wide range of frequencies. It is obvious that more transmission zeros occurred when more Moore-1 are united. To be exactly, the transmission zeros are proportional to the number of basic element. It is worth to point out that the influence of different occupied center area to the excitation is negligible which has been validated in [7]. Consequently, the resultant multitransmission zeros can be successfully interpreted through the self-similarity nature of fractals. This can be strongly supported by the results of microstrip resonators of conventional rectangle CRR occupying the same area with Moore-5. Nothing shows any evidence of such obvious multiband behavior which should be highlighted. Another most important features lie in two aspects, that is, the higher operation order is, the lower transmission zeros and the steeper transition between the passband and the primary transmission zero become.

Detailed multi resonance behavior of these MTM cells is summarized in Table 1. We obtain that the first transmission zero is in rigorous proportion to the number of Moore1 regardless of any stochastic error of simulation engine. That is to say, the first transmission zero of Moore- $n$-loaded microstrip MTM cells with the same minimal fractal segment dimension can be predicted as

$$
f_{\text {zero-1 }}^{\text {Moore } n}=f_{\text {zero-1 }}^{\text {Moore-1 }} / n \text {. }
$$

Concerning the second or even the higher transmission zeros, although these conditions are with much complexity and stochastic uncertainty, the conclusion of scale effect (4) 


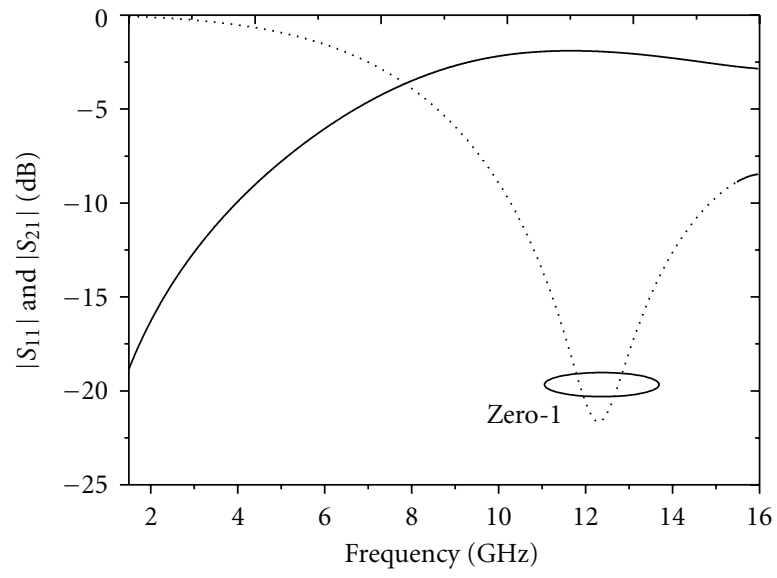

(a)

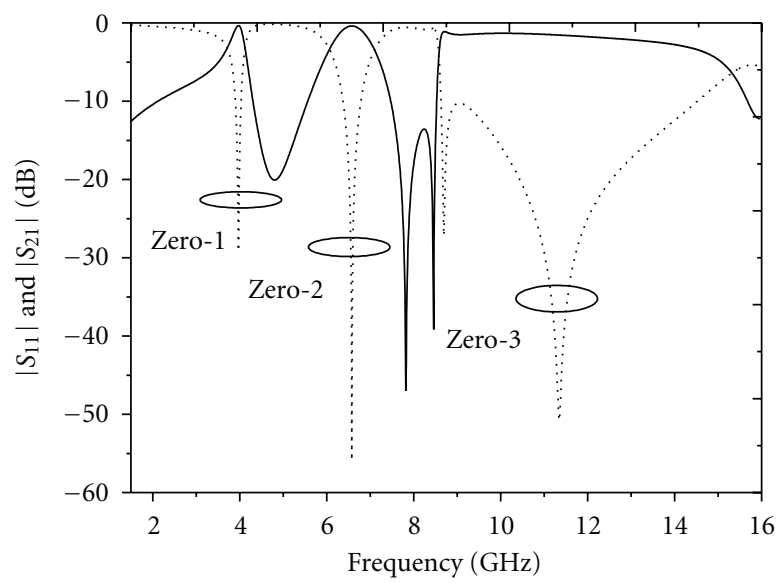

(c)

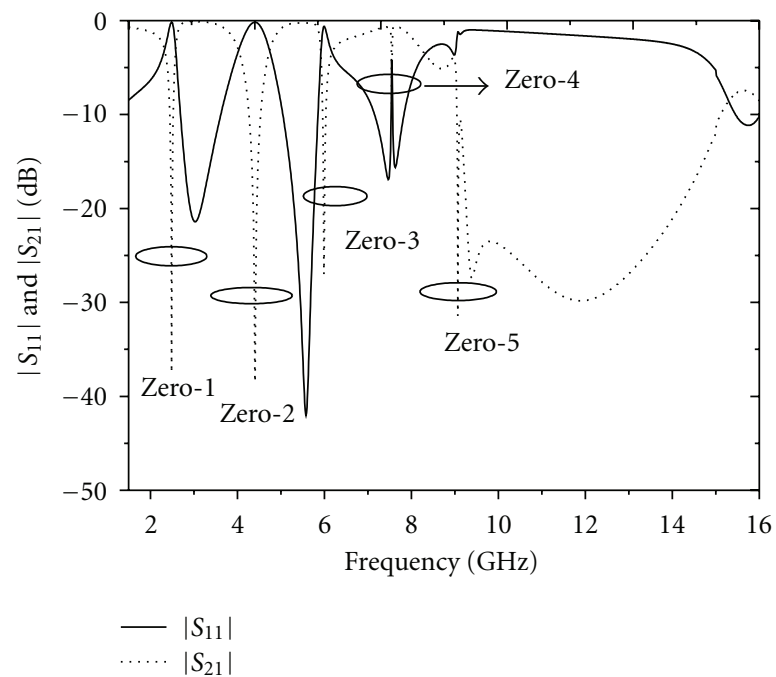

(e)

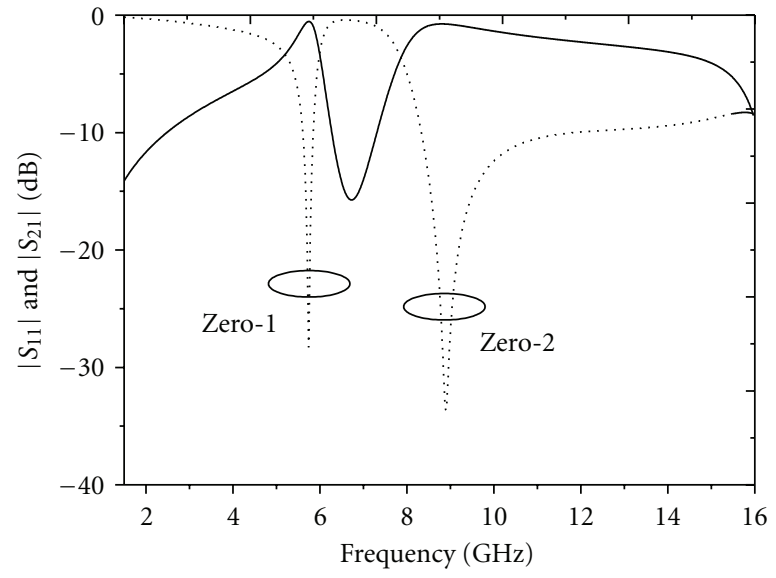

(b)

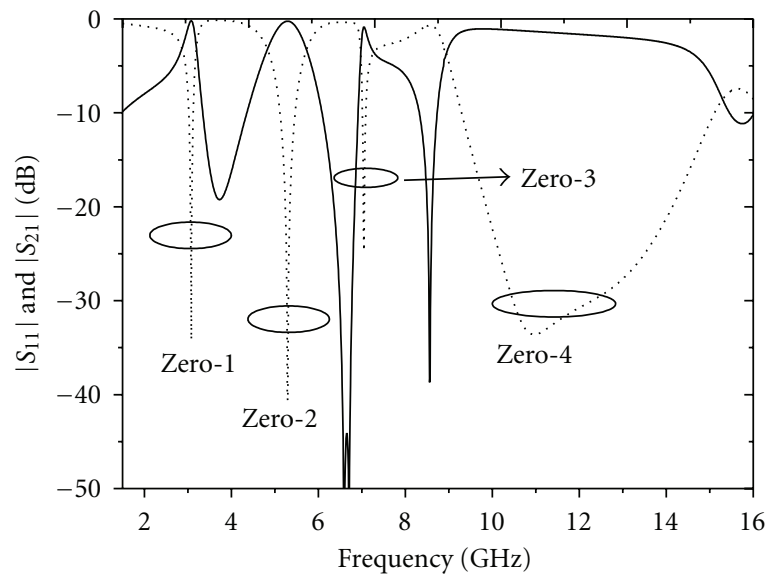

(d)

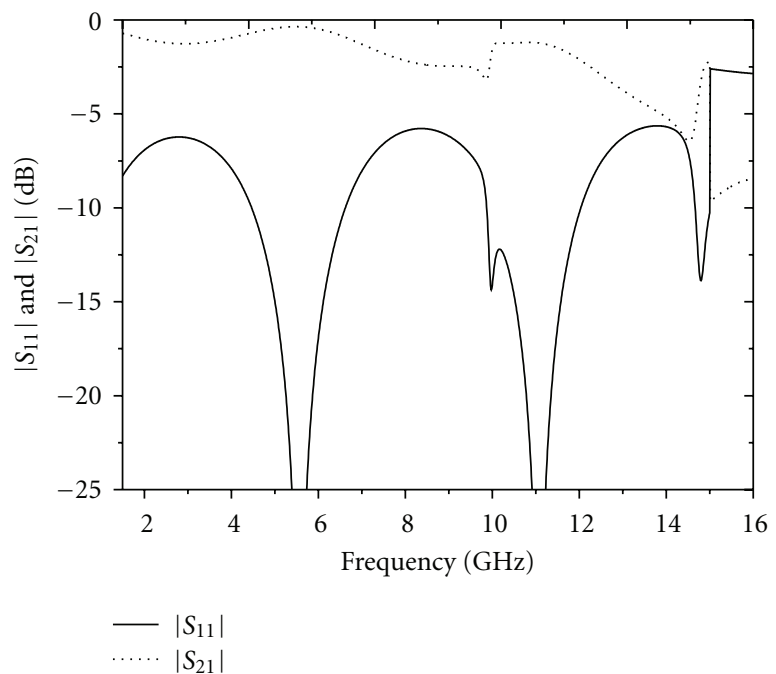

(f)

Figure 4: S-parameters of the proposed microstrip MTM cells based on (a) Moore-1, (b) Moore-2, (c) Moore-3, (d) Moore-4, (e) Moore-5, and (f) conventional CRR occupying the same area with Moore-5. 


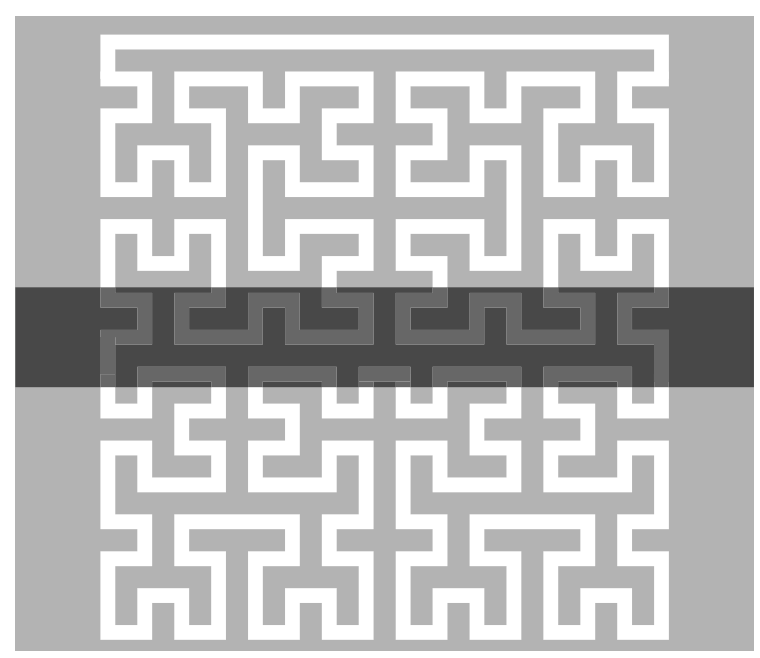

Figure 5: Microstrip MTM unit cell using H-CRR of Hilbert-4. Note that the H-CRR (depicted in white) is etched in the ground plane (depicted in light grey) underneath the host microstrip line (depicted in dark grey).

is still appropriate for them. As a consequence, we popularize (4) to derive the resonance of zero- $k$ which reads

$$
f_{\text {zero- }-k}^{\text {Moore-n }}=m / n f_{\text {zero- } k}^{\text {Moore- } m} \text {. }
$$

Note that the inequation of $n \geq m \geq k$ should be compulsorily satisfied.

2.3. Unit Cells That Use H-CRR. Figure 5 depicts the topology of MTM unit cell based on H-CRR of Hilbert-4. Note that this type of subwavelength particles is asymmetrical in the vertical direction of propagation. The proposed MTM cells are also built on the same substrate with former cells using M-CRR. Therefore identical physical parameters of microstrip line can be obtained except for the length which mainly depends on the structure of H-CRR. To meet the effective homogeneous requirement and simultaneously guarantee the quasi-TEM mode transmission, the de-embedding technique is applied and the dimension of microstrip line approximately equals to $\mathrm{H}$-CRR but larger than it. The dimensions are obtained $10 \mathrm{~mm}, 18 \mathrm{~mm}$, and $20 \mathrm{~mm}$ for Hilbert-1, Hilbert-2, and Hilbert-4, respectively. For comparison convenience, length and width of the minimal fractal segment of H-CRR are set to be $1 \mathrm{~mm}$ and $0.4 \mathrm{~mm}$. For demonstration, Ansoft Designer is applied to examine the H-CRR-loaded MTM cells.

In Figure 6, S-parameters are obtained from simulation of MTM unit cells of different operation in a wide range of frequencies. As can be observed, very obvious three transmission zeros are achieved for Hilbert- 1 while six for Hilbert2 and twelve for Hilbert-4. It shows that the multiband effect strengths and is proportional to the number of combined Hilbert-1. The conclusion derived from M-CRRloaded MTM unit cells can be straightforwardly applied to H-CRR-loaded ones. The only discrepancy lies in three is only one cardinal transmission zero in the former basic element of Moore-1 while three transmission zeros in the latter case. For comparison, the S-parameters of conventional counterpart of Hilbert-4 occupying the same area without fractal perturbation is also provided. Although four transmission zeros appear, they comprise higher harmonics induced by the periodic nature of transmission line not by multi resonance of fractals. In addition, a significant lower primary resonance $1.8 \mathrm{GHz}$ of Hilbert- 4 can be acquired with regard to its conventional counterpart whose primary transmission zero occurred at 5.2 GHz. As a consequence, the fractal perturbation results in a significant miniaturization of MTM cells which allows the design of more compact subwavelength particles.

Detailed multiband behavior of H-CRR-loaded MTM cells is summarized and provided in Table 2 . It can be found that the $k$ th resonance of Hilbert- $n$ can be roughly obtained by $k$ th resonance of Hilbert- $m$

$$
f_{\text {zero }-k}^{\text {Hilbert }-n}=m / n f_{\text {zero }-k}^{\text {Hilbert }-m} .
$$

Note that $k \leq 3 m$ and $m \leq n$ should be fulfilled. It is worth to point out that the computation of former resonances of these MTM cells may be with relatively larger error using (6), which can be successfully explained through that the perimeter of Hilbert- $n$ is not in rigorous proportion to Hilbert- $m$ by scale of $n / m$ like above-mentioned Moore fractals. However, with higher working frequency, the error caused by this effect is suppressed.

2.4. EM Field Distribution. To our knowledge, proper excitation of an inclusion or a ring is a very important issue in the fabrication of LH or other related single negative MTMs. Split ring resonators (SRR) [18] and omega-like inclusions [19] are excited by axial $\mathrm{H}$-field and thus exhibit negative permeability whereas inversely an axial E-field is excited in their complementary counterparts including CSRRs and complementary omega-like structure [20]. In general, it is useful to note that $\mathrm{H}$-field should be applied parallel to the axial direction of the rings or inclusions for magnetically driven resonator while inversely E-field for the electrically driven one.

To provide a deep insight into the characteristic and working mechanism of the proposed resonator, the Moore4 and Hilbert- 4 based MTM particles are investigated from field distribution angle. At this point, we would like to mention that residual MTM cells are also investigated. Since Moore- 4 and Hilbert- 4 are with much complexity and abundant conditions which contain the residual types, the results of them are completely representative.

The E-field and H-field distribution comparison of Moore-4-loaded MTM cell at different frequencies are displayed in Figure 7. It is characterized by the commercial finite-element based EM solver of Ansoft HFSS 11.0. Simulated S-parameters between Ansoft HFSS and Ansoft Design are in reasonable agreement, which has proved the effectiveness of the results. Since fringe field is less important for excitation, we focus our intention on the EM phenomenon in the center of Moore-4. At 3.07 and $5.22 \mathrm{GHz}$, very clear superiority of axial $\mathrm{H}$-field compared with E-field can be 
TABLE 1: Multi resonance comparison of MTM unit cells using different M-CRR.

\begin{tabular}{lcccccc}
\hline Operation & Transmission zeros & Zero-1 $(\mathrm{GHz})$ & Zero-2 $(\mathrm{GHz})$ & Zero-3 $(\mathrm{GHz})$ & Zero-4 $(\mathrm{GHz})$ & Zero-5 $(\mathrm{GHz})$ \\
\hline Moore-1 & 1 & 12.3 & - & - & - & - \\
Moore-2 & 2 & 5.75 & 8.91 & - & - \\
Moore-3 & 3 & 3.97 & 6.58 & 11.3 & - & - \\
Moore-4 & 4 & 3.08 & 5.29 & 7.05 & 10.9 & - \\
Moore-5 & 5 & 2.49 & 4.41 & 5.99 & 9.55 \\
\hline
\end{tabular}

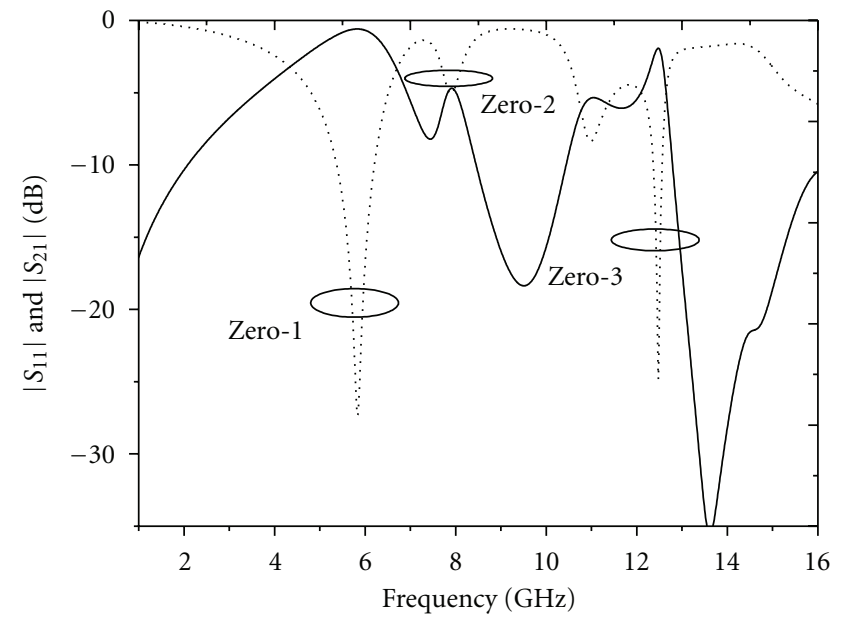

(a)

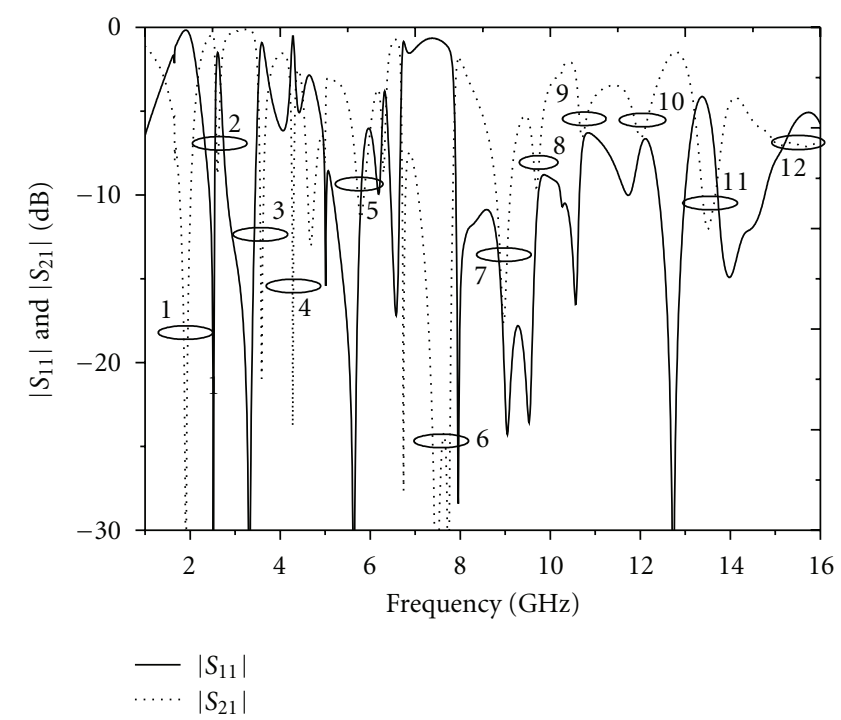

(c)

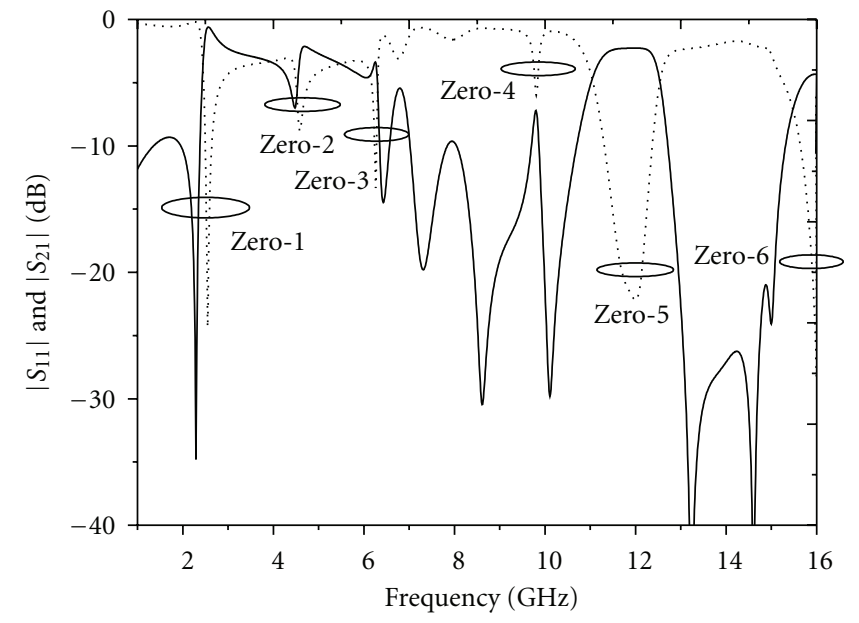

(b)

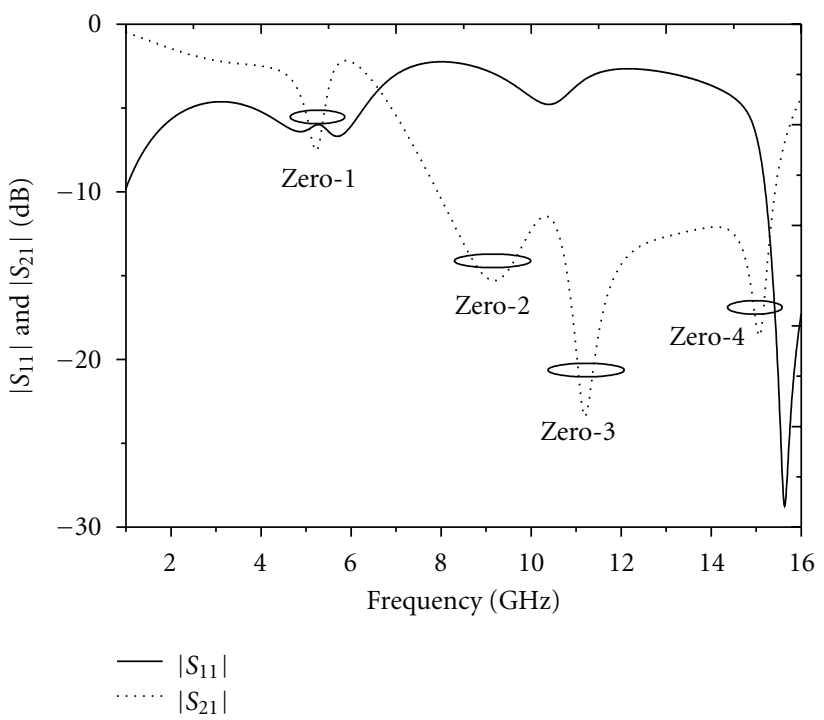

(d)

FIGURE 6: S-parameters of the MTM unit cell. (a) Hilbert-1. (b) Hilbert-2. (c) Hilbert-4. (d) Conventional CRR occupying the same area with Hilbert-4.

TABLE 2: Multi resonance comparison of MTM unit cells using different H-CRR.

\begin{tabular}{lccccccc}
\hline Operation & Transmission zeros & Zero-1 $(\mathrm{GHz})$ & Zero-2 $(\mathrm{GHz})$ & Zero-3 $(\mathrm{GHz})$ & Zero-4 $(\mathrm{GHz})$ & Zero-5 $(\mathrm{GHz})$ & Zero-6 $(\mathrm{GHz})$ \\
\hline Hilbert-1 & 3 & 5.86 & 7.91 & 12.49 & - & - & - \\
Hilbert-2 & 6 & 2.6 & 4.58 & 6.27 & 9.81 & 12.0 & 16 \\
Hilbert-4 & 12 & 1.8 & 2.6 & 3.59 & 4.28 & 5.8 & 7.7 \\
\hline
\end{tabular}




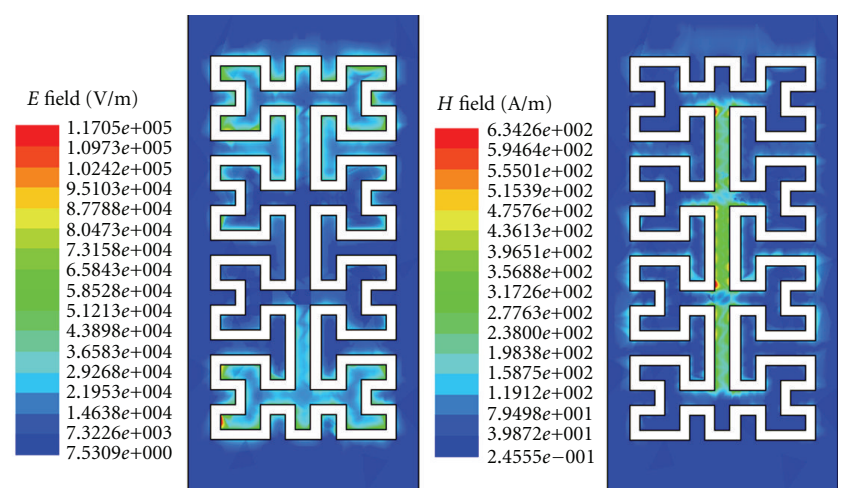

(a)

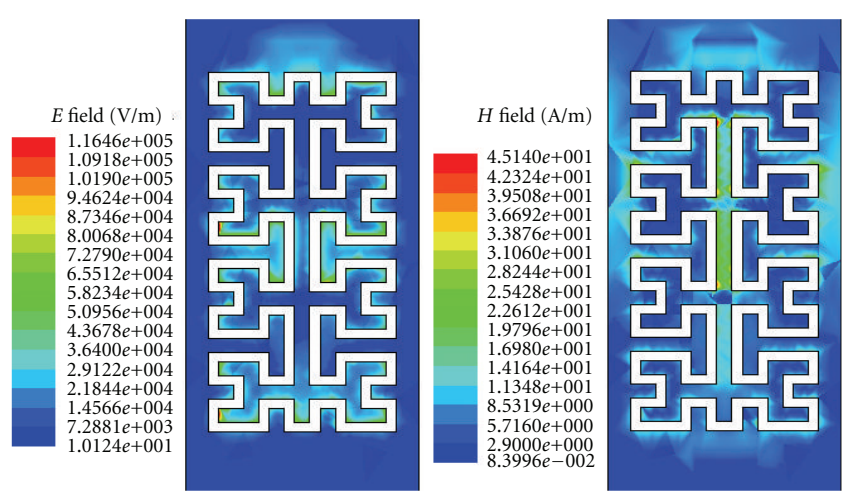

(b)

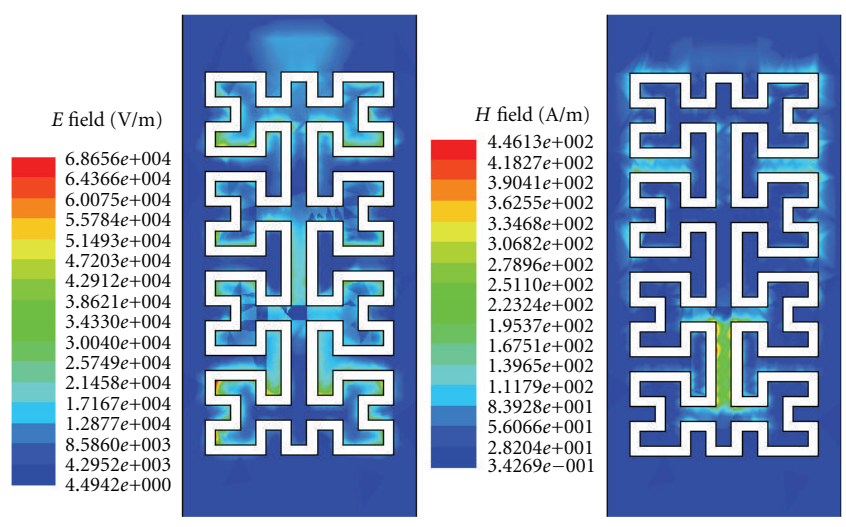

(c)

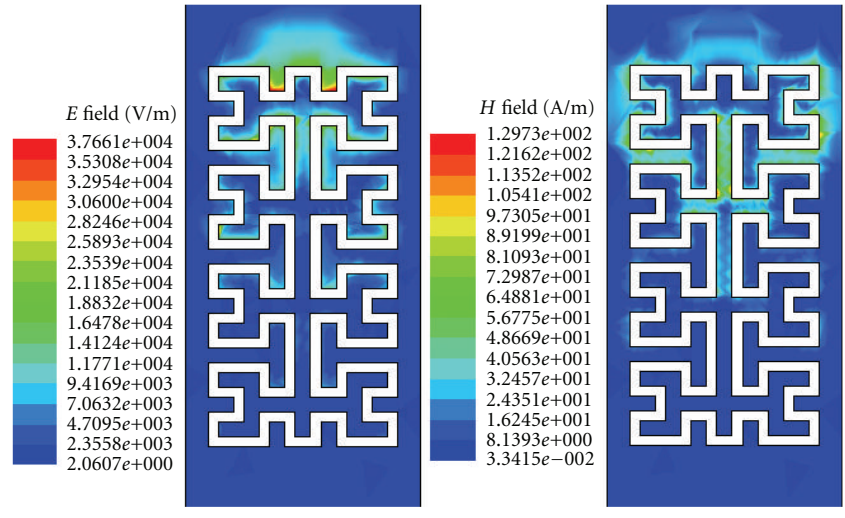

(d)

Figure 7: E-field and H-field distribution comparison of Moore-4-loaded MTM unit cell at (a) 3.07 GHz, (b) $5.02 \mathrm{GHz}$, (c) 7.05 GHz, and (d) $10.9 \mathrm{GHz}$.
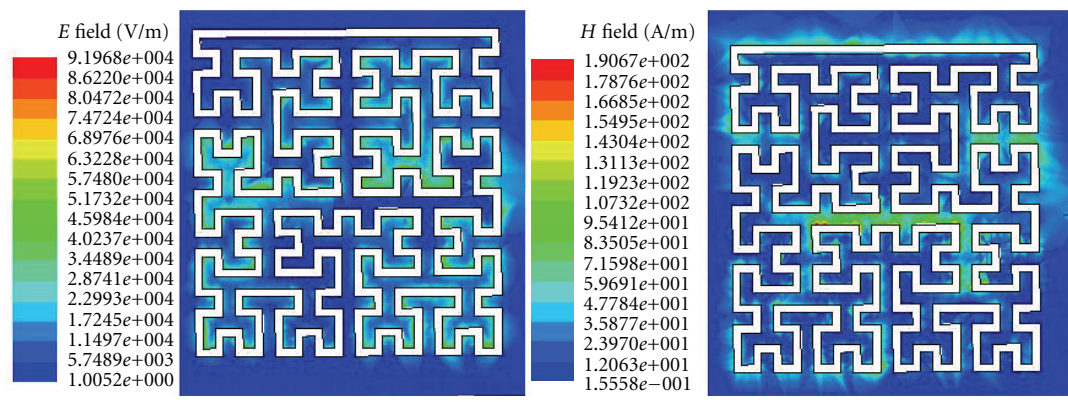

FIGURE 8: E-field and H-field distribution comparison of Hilbert-4-loaded MTM unit cell at 1.8 GHz.

observed. At $10.9 \mathrm{GHz}$, although the superiority of axial $\mathrm{H}$-field is less visible with regard to the first two cases, it is still with predominantly magnetic response. In spite of this, we should emphasize that the superiority of $\mathrm{H}$ field does not mean that there is no E-field excited parallel to the axial of CRR but with effort less than $\mathrm{H}$-field. As to $7.05 \mathrm{GHz}$, completely reversed superiority of E-field is indicated. Integrated with the knowledge and conclusion that have been drawn, we predict negative- $\mu$ would occur in the vicinity of zero- 1 , zero- 2 , and zero- 4 while negative- $\varepsilon$ beside zero-3.
In order to provide further insight into the operation mechanism of $\mathrm{H}$-CRR, the field distribution of Hilbert4-loaded MTM unit cell is also provided (see Figure 8). Very obvious superiority of axial $\mathrm{H}$-field to E-field at primary resonance $1.8 \mathrm{GHz}$ can be seen. In other words, the structure can be considered as magnetic-derived particle. Note that although the fringe E-field is superior to the $\mathrm{H}$ field at the first resonance, it contributes less to negative- $\varepsilon$. Consequently, behavior of negative- $\mu$ would exhibit around $1.8 \mathrm{GHz}$, which will be demonstrated in Section 3 through constitutive EM parameters. 


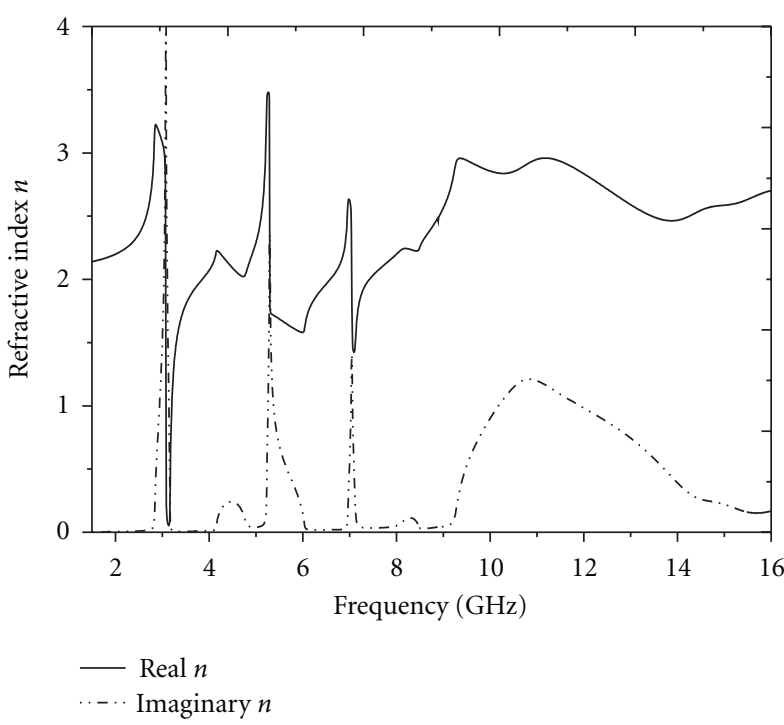

(a)

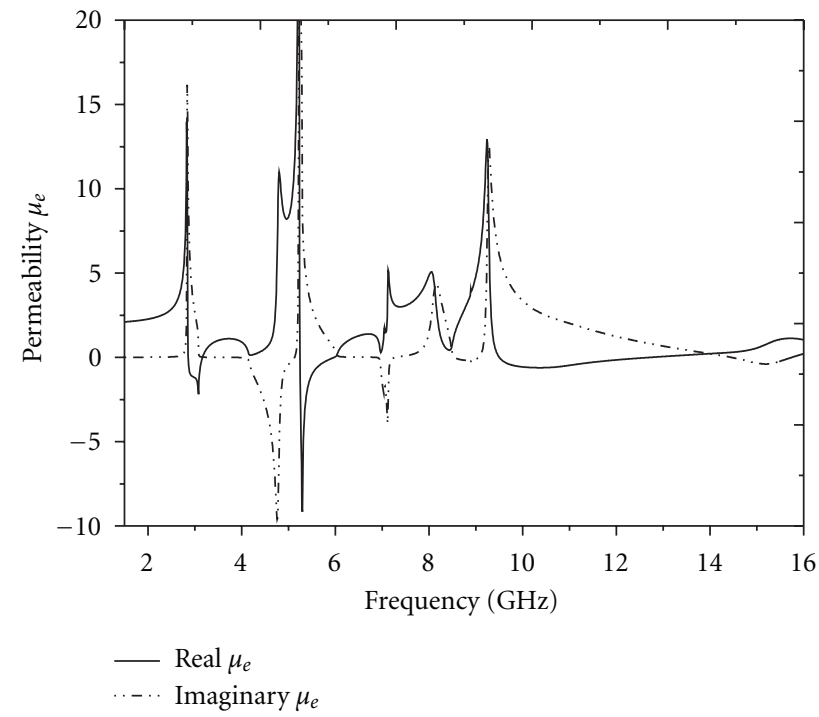

(b)

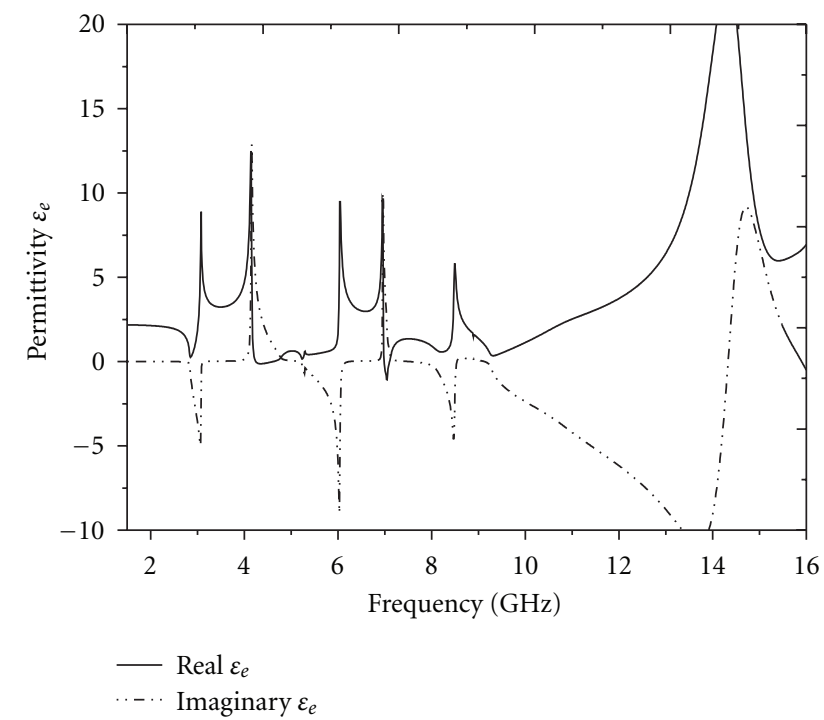

(c)

Figure 9: Extracted effective constitutive EM parameters of the Moore-4-loaded microstrip resonator. (a) Refractive index. (b) Effective permeability. (c) Effective permittivity.

\section{Effective EM Parameters}

Circuit modeling method is used as an efficient strategy in analysis of CSRRs [3] or SRR-loaded structure. As to the CRR, it is an easy task to derive the equivalent circuit model regarding the fundamental frequency band. However, we should have in mind these considerations, any model would suffer a severe degradation in describing the EM behavior when the frequencies extended to a wide range. That is to say, a more complicated circuit model should be developed to meet the expansion of frequency band. Fractal perturbation in CRR makes the condition more complicated even regardless of the harmonics. It is in the mechanism of performance repetition due to the self-similarity nature of fractals. As a consequence, modeling method would be inefficient and needless in dealing with the tough matter. In view of them, an improved constitutive EM parameters retrieval approach based on NRW (Nicolson-Ross-Weir) developed in [21] is applied as a substitution of modeling analysis.

Figure 9 shows the effective constitutive EM parameters of the Moore-4-loaded MTM unit cell. As expected, the negative $\mu_{e}^{\prime}$ exists in the vicinity of $3.07 \mathrm{GHz}, 5.22 \mathrm{GHz}$ and $10.9 \mathrm{GHz}$ while negative $\varepsilon_{e}^{\prime}$ around $7.05 \mathrm{GHz}$. Note that $\varepsilon_{e}^{\prime}$ and $\mu_{e}^{\prime}$ are the real part of effective permittivity and permeability, respectively. Thus a close correspondence between Figures 9 and 7 is achieved. In particular, the negative refractive index (NRI) does not exhibit first midst and last attributing to a single negative permittivity or permeability. In addition, the common negative $\varepsilon_{e}^{\prime}$ of CSRRs 


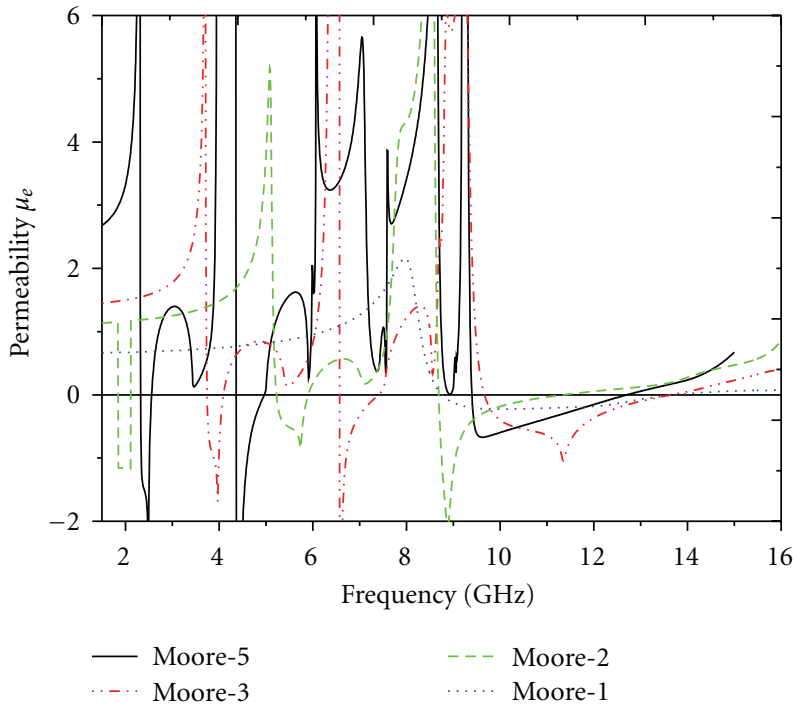

(a)

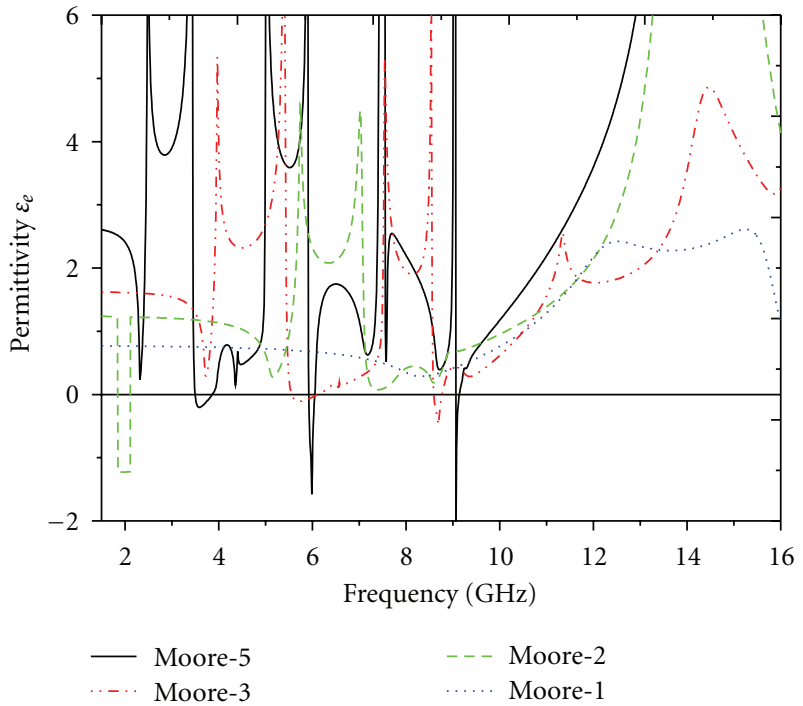

(b)

FIGURE 10: Comparison of effective constitutive EM parameters of the M-CRR-loaded microstrip resonators. (a) Real part of effective permeability. (b) Real part of effective permittivity.

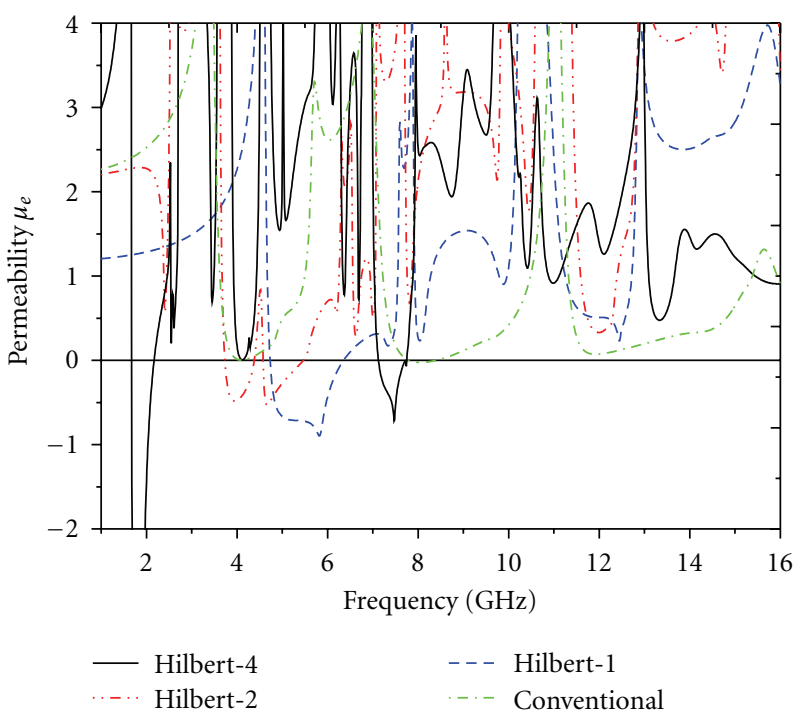

(a)

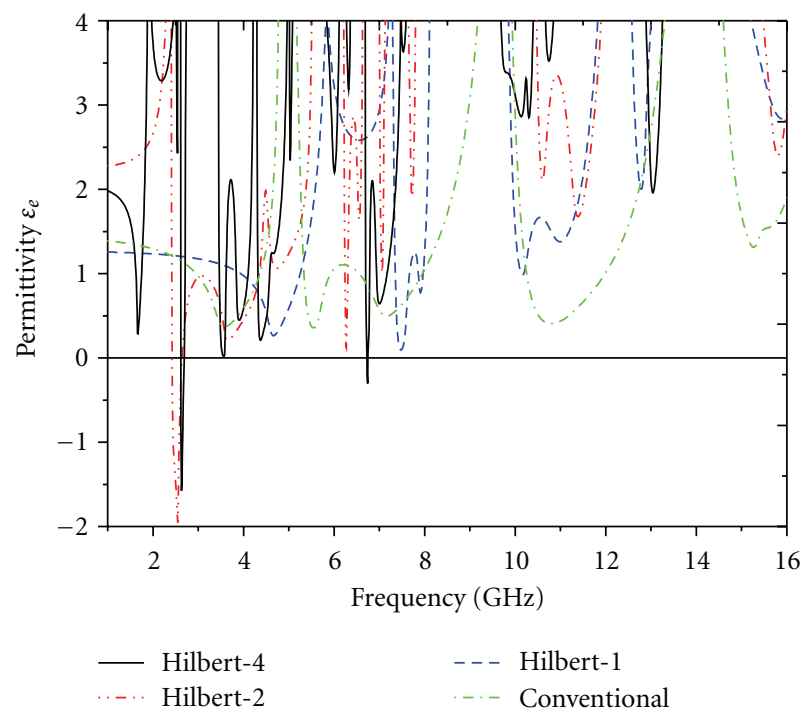

(b)

FIGURE 11: Comparison of effective constitutive EM parameters of the H-CRR-loaded MTM unit cells. (a) Real part of effective permeability. (b) Real part of effective permittivity.

is substituted by the negative $\mu_{e}^{\prime}$ of CRR for the primary transmission zero. It is the absence of separation gap in the CRR that causes the different operation mechanism.

The comparison of effective parameters of residual MCRR-loaded microstrip resonators is shown in Figure 10. As can be seen, the resultant transmission zero-1, zero-2, and the final transmission zero- $n$ are due to the negative $\mu_{e}^{\prime}$ while zero- 3 is attributed to the negative $\varepsilon_{e}^{\prime}$ for all cases. Note that the zero-3 is classified in final transmission zero for Moore-3. As to the zero- 4 of Moore-5, it can be successfully explained through the sharply increased $\varepsilon_{e}^{\prime \prime}$. Note that $\varepsilon_{e}^{\prime \prime}$ and $\mu_{e}^{\prime \prime}$ are the imaginary part of effective permittivity and permeability, respectively, and are corresponding to the electric and magnetic losses. It is obvious that negative $\mu_{e}^{\prime}$ for zero- 1 is controllable by adjusting the dimension.

Slightly different with the working mechanism of MCRR-loaded MTM cells, the primary transmission zeros of H-CRR-loaded MTM cells is with much complexity (see Figure 11). Despite this, both M-CRR- and H-CRR-loaded particles are with much resemblance. The negative $\mu_{e}^{\prime}$ occurs around the first transmission zero of Hilbert-1, Hilbert-4, second transmission zero of Hilbert-2, and sixth 


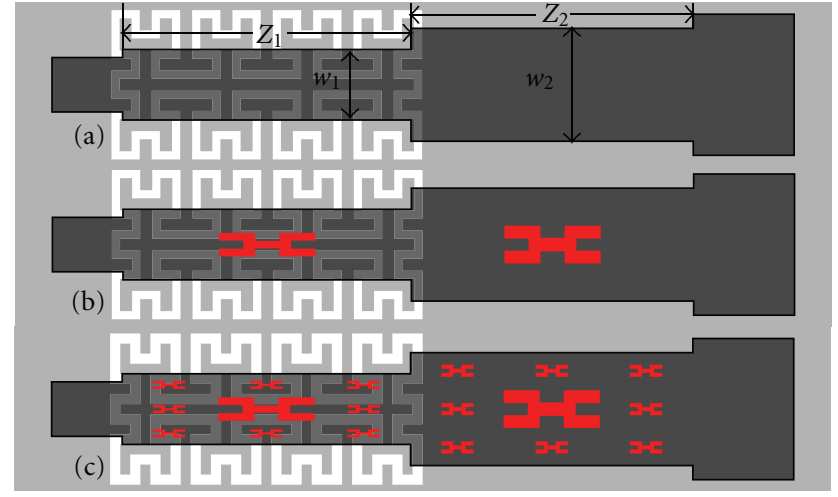

FIGURE 12: Topology of two-section SITs with Moore-4 (depicted in white) etched in the ground plane (depicted in light grey) and (a) SK0, (b) SK1 (depicted in red), and (c) SK2 on the conductor strip (depicted in dark grey).

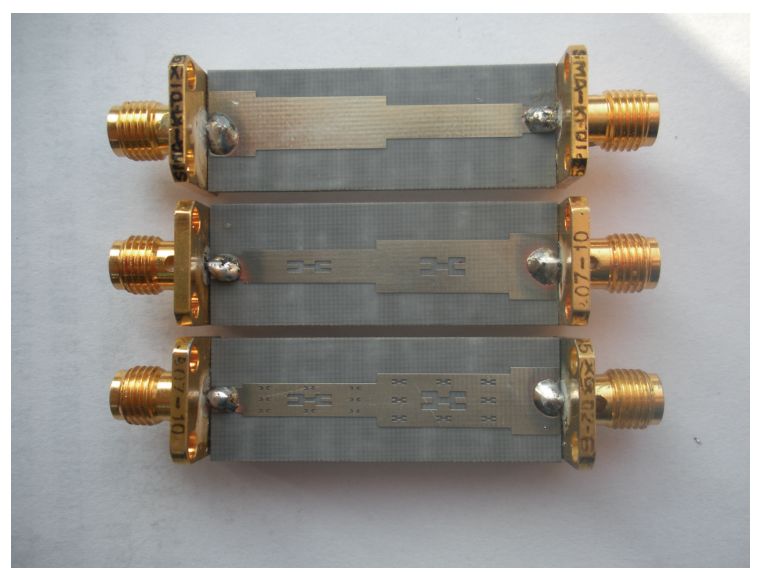

FIGURE 13: Fabricated prototype of the designed SITs.

transmission zero of Hilbert-4. The negative $\varepsilon_{e}^{\prime}$ happens in the vicinity of the first transmission zero of Hilbert-2, and the second transmission zero of Hilbert-4. The residual transmission zeros are attributing to the sharply increased $\varepsilon_{e}^{\prime \prime}$ or $\mu_{e}^{\prime \prime}$. However, there is no evidence showing negative $\mu_{e}^{\prime}$ or $\varepsilon_{e}^{\prime}$ in conventional CRR case. In other words, the single negative permittivity or permeability and multiresonances behavior are unique features of fractal-shaped CRR not of conventional one.

In general, results of Figures 9, 10, and 11 provide us a brand-new picture which would be a strong concussion to the conventional conception. Novel conclusions can be emphasized in following aspect. There is no consecutive negative $\varepsilon_{e}^{\prime}$ or $\mu_{e}^{\prime}$ for the fractal perturbed CRR. By contraries, the negative $\varepsilon_{e}^{\prime}$ and $\mu_{e}^{\prime}$ even occurs by turns in some cases, which is essentially induced by the alternately changed superiority of axial E-field and $\mathrm{H}$-field. That is to say, the fractalshaped CRR is electrically derived in some bands with negative $\varepsilon_{e}^{\prime}$ and magnetically derived in other bands with negative $\mu_{e}^{\prime}$.

\section{Design Examples}

To illustrate the potential application of the proposed MCRR- and H-CRR-loaded MTM unit cell to the passive devices with the requirement of high selectivity, two types of prototype device examples are provided. The first type is the two-section maximal flat SIT switching from $50 \Omega$ to $25 \Omega$. The other type is the Hi-Lo microstrip LPF.

4.1. M-CRR-Based SITs Using Hybrid Prefractal Shape. SIT, which allows the available input power transferred from one stage to the next smoothly, is one of the most fundamental devices in microwave and RF circuits. Recently, we have found that fractal perturbation in the low impedance sections of UWB filter results in improved passband performance and sharpened transition [12]. The design concept is extended directly to this work including the followed LPF implementation. It is exploited in terms of impedance bandwidth enhancement and will be demonstrated to be a very effective strategy in this work. Enlightened by the integrated concept [13], the M-CRR of Moore-4 is considered to integrate with the SIT to introduce additional selectivity.

For quarter-wavelength SITs, two- or three-section maximally flat SIT is preferably considered for practical application in virtue of good performance and appropriate circuit size. For $n=2$, and $n=3$, the junction VSWR $V$ of maximally flat quarter-wavelength SIT is associated with the following [22]:

$$
\begin{gathered}
V_{1}=\rho^{1 / 4}, \\
V_{2}=\rho^{1 / 2}, \\
V_{1}^{2}+2 \rho^{1 / 2} V_{1}-\frac{2 \rho^{1 / 2}}{V_{1}}-\frac{\rho}{V_{1}^{2}}=0, \\
V_{2}=\rho^{1 / 2} / V_{1},
\end{gathered}
$$

where $\rho$ is impedance ratio of the load $\left(Z_{L}\right)$ and source $\left(Z_{S}\right)$ impedance. Values of residual $V$ are achieved from the symmetry relations provided by

$$
V_{i}=V_{n+2-i} .
$$

With the determined $V_{i}$, normalized impedance $Z_{i}$ of the $i$ th section referred to the minimum of $Z_{S}$ and $Z_{L}$ can be obtained by solving the following equations:

$$
\begin{gathered}
V_{i}=\frac{1+\Gamma_{i}}{1-\Gamma_{i}}, \\
\Gamma_{i}=\frac{Z_{i}-Z_{i-1}}{Z_{i}+Z_{i-1}} .
\end{gathered}
$$

Note that $Z_{S}$ is also referred as $Z_{0}$ in (9). For experiment in this work, the implementation of two-section SITs operating at $3.5 \mathrm{GHz}$ are performed on F4B-2 substrate with $1 \mathrm{~mm}$ thick thickness, and dielectric constant of 2.65. The load and source impedance are set to be $Z_{L}=25 \Omega$ and $Z_{S}=50 \Omega$ respectively. Physical parameters of conventional two-section SITs are obtained as $Z_{1}=42 \Omega, Z_{2}=29.7 \Omega, L_{1}=14.2 \mathrm{~mm}, L_{2}$ $=14 \mathrm{~mm}, w_{1}=3.5 \mathrm{~mm}$, and $w_{2}=5.7 \mathrm{~mm}$, respectively. With 


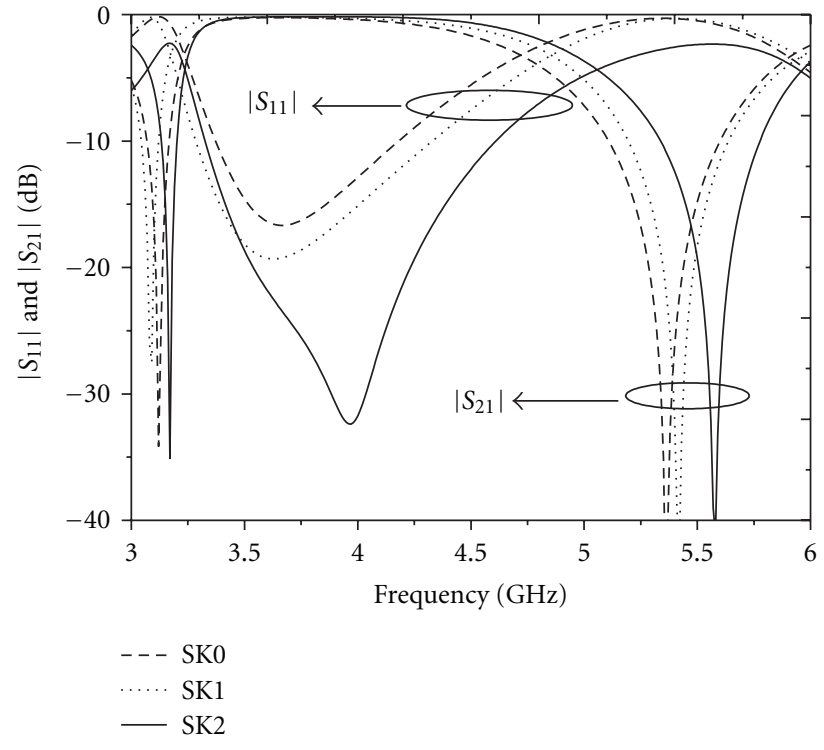

(a)

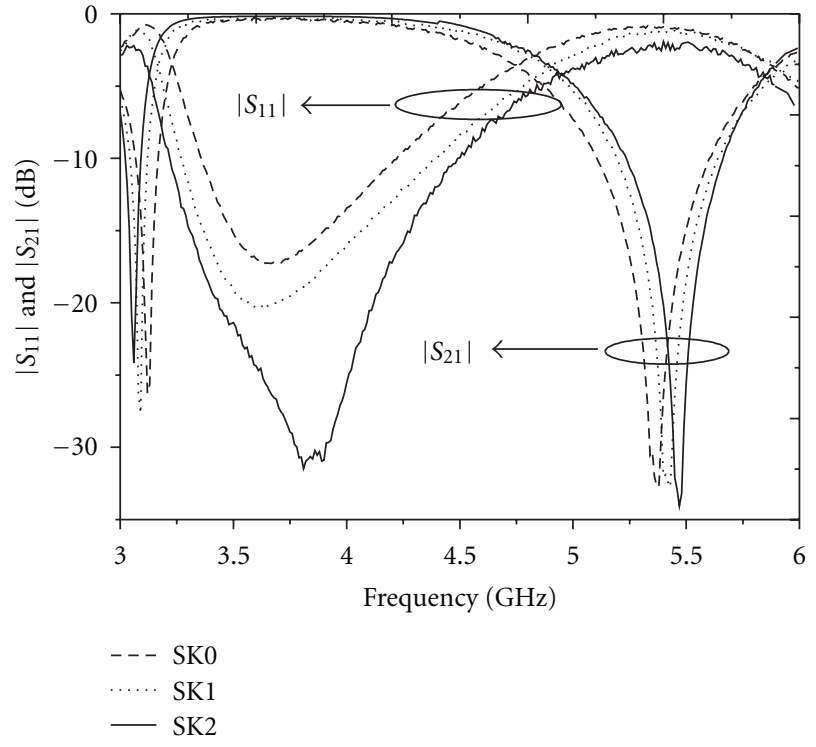

(b)

FIGURE 14: Frequency response of the proposed SITs based on SK0, SK1, and SK2, respectively. (a) Simulation. (b) Measurement.

determined dimensions of conventional SITs, the following step is considered to introduce M-CRR to improve the outof-band selectivity.

Figure 12 shows the topology of three types of SITs incorporating M-CRR. The M-CRR with two edged transmis-sion zeros functions a bandpass filter in the current application. Once the shape of fractals is selected, the next step is engineered to locate the operating frequency between the resultant two attenuation poles by carefully tailoring the dimension of M-CRR. Since the introduced M-CRR is demonstrated without significant influence on the impedance of microstrip line if provided proper location, thus it can be directly used in SIT just by tiny optimization of the loaded position. Moore- 4 with minimum fractal seg-ment of $1 \mathrm{~mm}$, which etched underneath the first quarter-wavelength stepped section, is considered in final SIT design.

To enhance the performances in terms of impedance match and bandwidth enhancement, the Sierpinski carpet of different iteration level is exploited on the conductor strip. In addition, Koch island of the first iteration order is applied instead of conventional rectangle as its initial generator from the point of better current continuity. For convenience, the presented hybrid structures of Koch island and Sierpinski carpet of zeroth, first, and second order are denoted as SK0, SK1, and SK2, respectively.

For demonstration, novel-designed two-section SITs are analyzed through Ansoft Designer. Analysis of conventional SIT is also provided from the point of comparison. For verification, they are fabricated and measured through the Anritsu ME7808A Vector Network Analyzer. Figure 13 shows the fabricated prototypes. Figure 14 exhibits the simulated and measured frequency response. It is found that the simulated and measured results are in reasonable agreement except slight frequency shift for measurement data of SK2. Nevertheless, this tiny discrepancy is in a normal level and is induced inherently in the fabrication process. From Figure 14, two clear attenuation poles locate in the lower and upper edge of passband and thus enable high selectivity of the SITs. Concentrate on impedance bandwidth of these SITs, we conclude that the $10 \mathrm{~dB}$ return-loss $\left(\left|S_{11}\right|\right)$ impedance bandwidth is evidently enhanced as iteration order increases. For instance, the measured bandwidth of $1.12 \mathrm{GHz}$ for SK1 has broadened by $30.2 \%$, and is $1.27 \mathrm{GHz}$ for SK2 by $47.7 \%$ with regard to $0.86 \mathrm{GHz}$ for conventional SK0. It is useful to declare, although the performance of return loss deteriorates to some degree attributing to the coupling between microstrip line and Moore-4, the obtained highly integrated circuit (BPF combined SIT) should be emphasized.

4.2. H-CRR-Based LPF Using Crown Square. Since Hilbert2 exhibits negative permittivity at primary resonance, it is a preferable candidate to improve the selectivity. For possible application, a novel six-section Hi-Lo microstrip LPF is presented based on Hilbert-2. The LPF is specified by the cutoff frequency of $2.5 \mathrm{GHz}$ and maximally flat Butterworth frequency response. Characteristic impedance of the highand low-impedance sections are set to be 120 and $15 \Omega$, respectively. The F4B-2 substrate with dialectic constant of 2.65 and $1 \mathrm{~mm}$ thickness is applied for current LPF design. Particular design procedures are described as follows.

The physical parameters of conventional Hi-Lo LPF can be obtained following the design equations of [22]. Then, construct the high impedance section in Koch curve of first iteration order to reduce the size. Key factor of this process is to maintain the phase of Koch-shaped microstrip line unchanged with regard to its conventional counterpart. The 


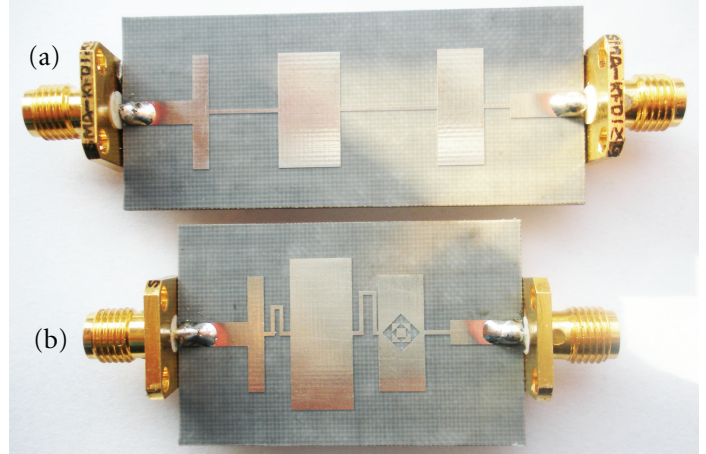

(A)

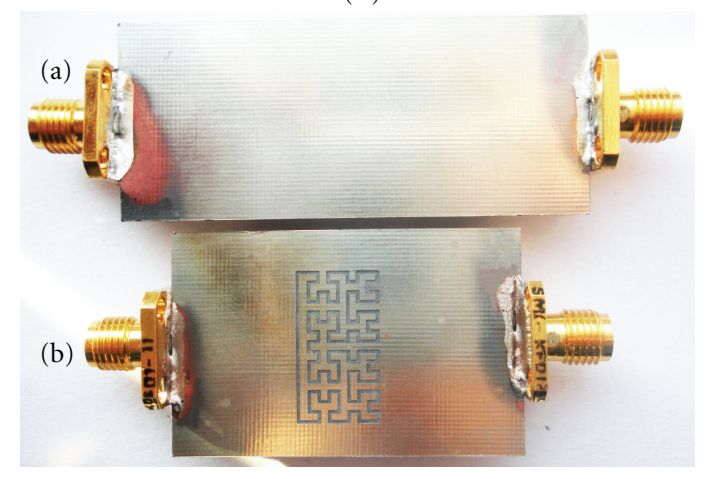

(B)

Figure 15: (A) Top view and (B) bottom view of the fabricated prototype of Hi-Lo microstrip LPFs. (a) Conventional design (b) Novel design with crown square.

resonance of proposed MTMs is controllable by the shape and dimension of CRR, therefore once the shape is fixed the residual assignment is to carefully tune the dimension of Hilbert-2 to locate the first resonance at the cutoff frequency, followed by a tiny adjustment of the placement of it. Hilbert2 with minimal fractal segment of $1 \mathrm{~mm}$ is etched under the second low impedance section in final LPF design. Width of the second low impedance section has been extended and equals to the dimension of Hilbert- 2 attributing to the resultant coupling between microstrip section and $\mathrm{H}$ CRR. Since the first low-impedance section is with width two narrow to be dug out any structure. Crown square perturbation in the second low-impedance part may lead to a shift of the primary resonance of CRR in virtue of affected coupling which in turn makes the LPF design more complex. Taking into account these factors, the next step is consisting in digging out a structure of crown square of first order from the third low-impedance section for this issue. Figure 15 depicts the fabricated prototypes of conventional and currently developed Hi-Lo LPF.

Novel designed LPF with or without crown square and its conventional counterpart are simulated through Ansoft Designer. Figure 16 illustrates the simulated fullwave S-parameters. Two most important aspects should be highlighted. First, the applied H-CRR has induced a significant steeper transition band. The selectivity of novel developed LPFs with or without crown square are calculated

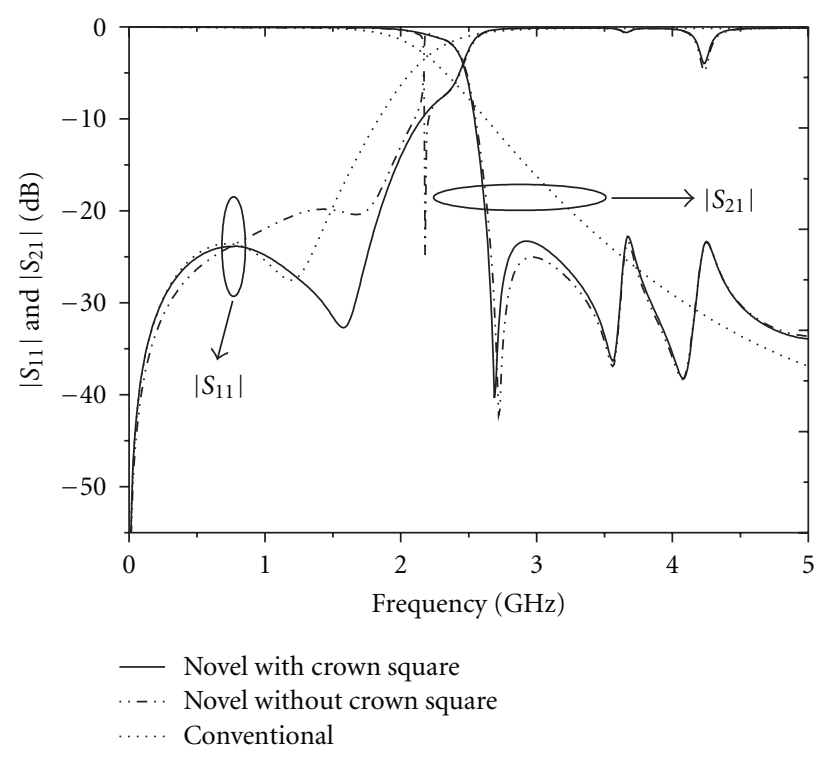

FIGURE 16: Comparison of simulated S-parameters of conventional LPF and novel designed LPF with or without crown square.

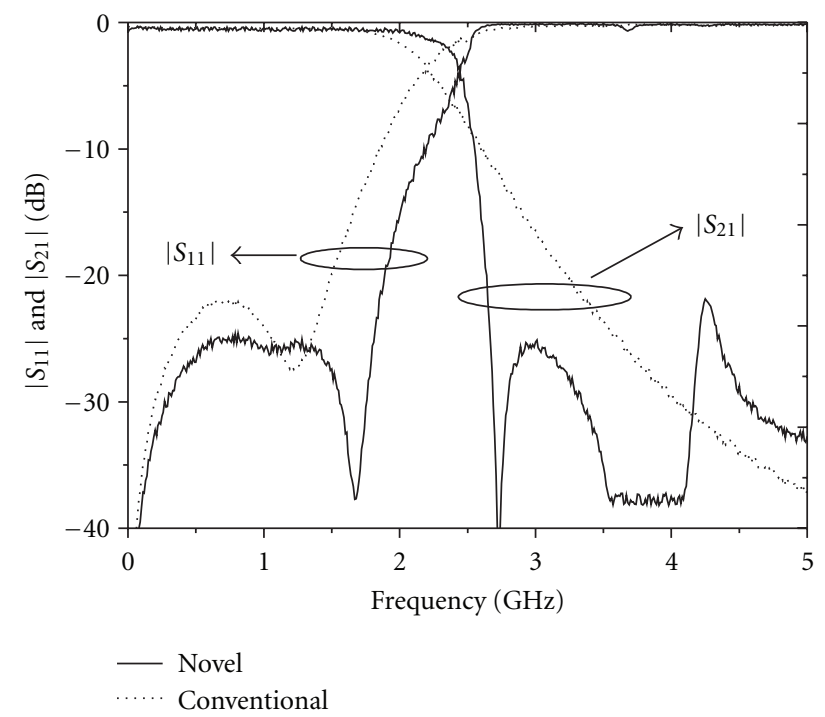

FIGURE 17: Comparison of measured S-parameters of conventional LPF and novel designed LPF with crown square.

as $89.4 \mathrm{~dB} / \mathrm{GHz}$ while conventional $\mathrm{LPF}$ as $15.7 \mathrm{~dB} / \mathrm{GHz}$. Second, the introduced crown square demonstrated has no influence on the stopband performance but results in significant improvement of passband performance, especially for the impedance bandwidth defined by $20 \mathrm{~dB}$ return loss, which has obviously extended from $1.46 \mathrm{GHz}$ (conventional LPF) to $1.85 \mathrm{GHz}$ (novel designed LPF with crown square).

For verification, the proposed LPF with crown square and its conventional counterpart are fabricated and measured. The measured data, characterized through Anritsu ME7808A vector network analyzer is compared in Figure 17. Consistent results between simulation and measurement can be observed in the entire frequency range of interest, which 
has confirmed the design concept. Measurement results reveal that proposed LPF exhibits almost flat insertionloss response approximating to $0.5 \mathrm{~dB}$, steep rejection skirt ranged from $2.42 \mathrm{GHz}$ to $2.64 \mathrm{GHz}$ (with selectivity of $77.3 \mathrm{~dB} / \mathrm{GHz}$ ), low sidelobe level of in-band return loss with maximal value reduced from $22 \mathrm{~dB}$ to $24.6 \mathrm{~dB}$, and obviously broadened impedance bandwidth (also defined by $20 \mathrm{~dB}$ return loss) from $1.49 \mathrm{GHz}$ to $1.88 \mathrm{GHz}$ (a scale of bandwidth enhancement by $26.2 \%)$. What is more, the prototype of proposed LPF obtained an additional 36.3\% size reduction relative to its conventional counterpart.

\section{Conclusion}

Novel single negative MTM TLs constructed by fractalshaped CRR are presented for the first time. The single negative permittivity or permeability has been demonstrated by constitutive EM parameters. Unlike conventional CSRRs featured invariable constitutive parameters, the proposed MCRR and H-CRR are with abundant character of single negative permittivity or permeability and even with them occurred by turns. For a deep insight, we find that whether a negative permittivity or permeability is shown mainly depends on whether the CRR is excited by superior axial E-field or H-field. Multiband behavior of these MTMs can be successfully interpreted through the self-similarity nature of fractals. Moreover, the unique behavior of negative constitutive parameters is only confined to fractal-shaped CRR.

For possible application, two prototype device examples are designed, fabricated, and measured. By integrating MCRR in the ground plane (functioned as a BPF), the SITs obtain a pair of attenuation poles (realized by negative permeability) in the lower and upper edge of passband and thus good selectivity is generated. By properly integrating $\mathrm{H}$-CRR (in virtue of negative permittivity), the selectivity of LPF $(77.3 \mathrm{~dB} / \mathrm{GHz})$ has been significantly improved by $77.1 \%$ with regard to its conventional counterpart $(15.8 \mathrm{~dB} / \mathrm{GHz})$ while without affecting passband performance. The introduced hybrid of Sierpinski carpet and Koch island in SITs, and Crown square in LPF have significantly broadened the impedance bandwidth (by a scale of $47.7 \%$ and $26.2 \%$, resp.) for the first time. The working principle of it is that the refined fractal perturbation in the low-impedance section has significantly suppressed the sharp current discontinuity caused by high-low-steps.

\section{Acknowledgment}

This work was supported by the National Natural Science Foundation of China under Grant no. 60971118.

\section{References}

[1] F. Falcone, T. Lopetegi, M. A. G. Laso et al., "Babinet principle applied to the design of metasurfaces and metamaterials," Physical Review Letters, vol. 93, no. 19, Article ID 197401, 4 pages, 2004.

[2] F. Falcone, T. Lopetegi, J.D. Baena, R. Marques, F. Martin, and M. Sorolla, "Effective negative- $\varepsilon$ stop-band microstrip lines based on complementary split-ring resonators," IEEE Microwave and Wireless Components Letters, vol. 14, no. 6, pp. 280282, 2004.

[3] J. D. Baena, J. Bonache, F. Martin et al., "Equivalent-circuit models for split-ring resonators and complementary split-ring resonators coupled to planar transmission lines," IEEE Transactions on Microwave Theory and Techniques, vol. 53, no. 4, pp. 1451-1461, 2005.

[4] M. Gil, J. Bonache, J. García-García, J. Martel, and F. Martín, "Composite right/left-handed metamaterial transmission lines based on complementary split-rings resonators and their applications to very wideband and compact filter design," IEEE Transactions on Microwave Theory and Techniques, vol. 55, no. 6, pp. 1296-1304, 2007.

[5] H. Y. Zeng, G.-M. Wang, C. X. Zhang, and L. I. Zhu, "Compact microstrip low-pass filter using complementary split ring resonators with ultra-wide stopband and high selectivity," Microwave and Optical Technology Letters, vol. 52, no. 2, pp. 430-433, 2009.

[6] A. Ali and Z. Hu, "Negative permittivity meta-material microstrip binomial low-pass filter with sharper cut-off and reduced size," IET Microwaves on Antennas and Propagation, vol. 2, no. 1, pp. 15-18, 2008.

[7] V. Crnojević-Bengin, V. Radonić, and B. Jokanović, "Fractal geometries of complementary split-ring resonators," IEEE Transactions on Microwave Theory and Techniques, vol. 56, no. 10, pp. 2312-2321, 2008.

[8] H. X. Xu, G. M. Wang, and C. X. Zhang, "Study and design of dual-band quarter-wave open-circuit stub based on Kochfractal-shaped geometry of CSRRs," in Proceedings of the IEEE International Conference on Microwave and Millimeter Wave Technology, pp. 1778-1780, Chengdu, China, May 2010.

[9] H. X. Xu, G. M. Wang, Q. Peng, and J. G. Liang, "Novel design of tri-band filter based on fractal shaped geometry of CSSRR," International Journal of Electronics, vol. 98, no. 5, pp. 647-654, 2011.

[10] H. X. Xu, G. M. Wang, C. X. Zhang, and Y. Hu, "Microstrip approach benefits quad splitter," Microwaves \& RF, vol. 49, no. 6, pp. 92-96, 2010.

[11] L. Yousefi and O. M. Ramahi, "Artificial magnetic materials using fractal hilbert curves," IEEE Transactions on Antennas and Propagation, vol. 58, no. 8, pp. 2614-2622, 2010.

[12] H. X. Xu, G. M. Wang, and C. X. Zhang, "Fractal-shaped UWB bandpass filter based on composite right/left handed transmission line," Electronics Letters, vol. 46, no. 4, pp. 285-286, 2010.

[13] H. Zhang and H. Xin, "A dual-band dipole antenna with integrated-balun," IEEE Transactions on Antennas and Propagation, vol. 57, no. 3, pp. 786-789, 2009.

[14] G. Peano, "Sur une courbe, qui remplit toute une aire plane," Mathematische Annalen, vol. 36, no. 1, pp. 157-160, 1890.

[15] J. McVay, N. Engheta, and A. Hoorfar, "High impedance metamaterial surfaces using Hilbert-curve inclusions," IEEE Microwave and Wireless Components Letters, vol. 14, no. 3, pp. 130-132, 2004.

[16] X. Chen, Y. Liu, and S. Safavi-Naeini, "Printed plane-filling fractal antennas for UHF band," in Proceedings of the IEEE Antennas and Propagation Society AP-S International Symposium, vol. 4, pp. 3425-3428, 2004.

[17] H. Ghali and T. A. Moselhy, "Miniaturized fractal rat-race, branch-line, and coupled-line hybrids," IEEE Transactions on Microwave Theory and Techniques, vol. 52, no. 11, pp. 25132520, 2004. 
[18] J. B. Pendry, A. J. Holden, D. J. Robbins, and W. J. Stewart, "Magnetism from conductors and enhanced nonlinear phenomena," IEEE Transactions on Microwave Theory and Techniques, vol. 47, no. 11, pp. 2075-2084, 1999.

[19] J. Huangfu, L. Ran, H. Chen et al., "Experimental confirmation of negative refractive index of a metamaterial composed of $\Omega$-like metallic patterns," Applied Physics Letters, vol. 84, no. 9, pp. 1537-1539, 2004.

[20] N. T. Messiha, A. M. Ghuniem, and H. M. El-Hennawy, "Planar transmission line medium with negative refractive index based on complementary Omega-like structure," IEEE Microwave and Wireless Components Letters, vol. 18, no. 9, pp. 575-577, 2008.

[21] H. X. Xu, G. M. Wang, and J. G. Liang, "Novel CRLH TL metamaterial and compact microstrip branch-line coupler application," Progress In Electromagnetics Research C, vol. 20, pp. 173-186, 2011.

[22] G. L. Matthaei, L. Young, and E. M. T. Jones, Microwave Filters, Impedance-Matching Networks, and Coupling Structures, Artech House, Norwood, Mass, USA, 1980. 

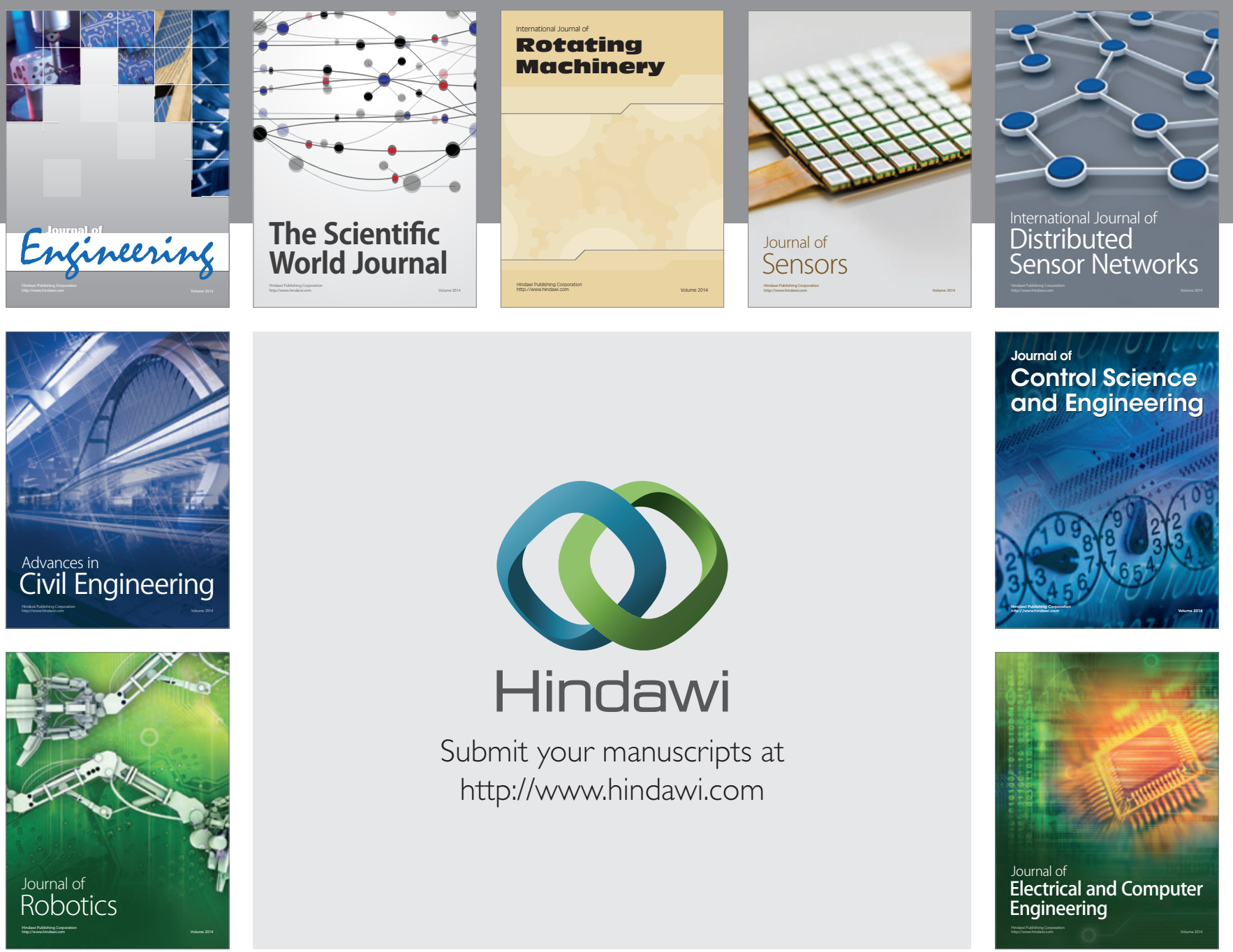

Submit your manuscripts at

http://www.hindawi.com
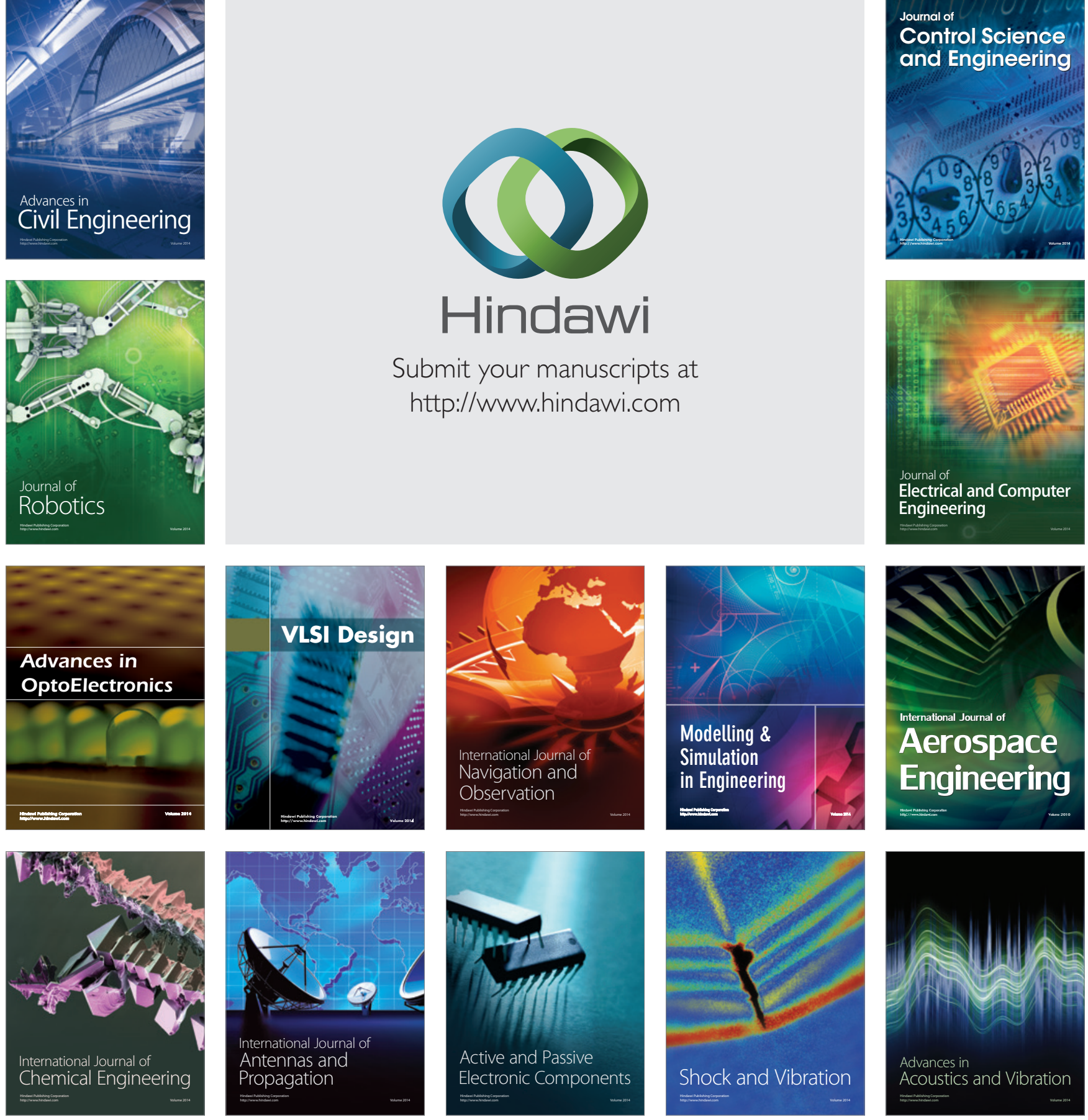